\title{
Cryogenically formed discharge waveguide
}

\author{
K. K. Swanson, ${ }^{1,2}$ A. J. Gonsalves $\odot,{ }^{1,}{ }^{*}$ H.-S. Mao $\odot,{ }^{3}$ T. Sipla, ${ }^{1}$ S. S. Bulanov $\odot,{ }^{1}$ N. A. Bobrova, ${ }^{3}$ \\ P. V. Sasorov $\odot,{ }^{3,4}$ G. Korn, ${ }^{4}$ C. Benedetti, ${ }^{1}$ C. V. Pieronek $\odot,{ }^{1,2}$ C. B. Schroeder $\odot,{ }^{1,2}$ \\ C. G. R. Geddes, ${ }^{1}$ E. Esarey, ${ }^{1}$ and W. P. Leemans $\circledast^{1,2,5}$ \\ ${ }^{1}$ Lawrence Berkeley National Laboratory, 1 Cyclotron Rd, Berkeley, California 94720, USA \\ ${ }^{2}$ University of California-Berkeley, Berkeley, California 94720, USA \\ ${ }^{3}$ Keldysh Institute of Applied Mathematics, Moscow 125047, Russia \\ ${ }^{4}$ Institute of Physics ASCR, v.v.i. (FZU), ELI-Beamlines, 18221 Prague, Czech Republic \\ ${ }^{5}$ Deutsches Elektronen Synchrotron, Notkestraße 8522607 Hamburg, Germany
}

(Received 16 October 2020; accepted 26 July 2021; published 20 September 2021)

\begin{abstract}
We describe the development and operation of a regenerative, cryogenically-formed discharge waveguide formed by freezing nitrous oxide gas onto the inner wall of a sapphire capillary. We demonstrate a technique for varying the channel diameter in situ and present guiding of low-power laser pulses through 6-cm long waveguides with channel diameters of $0.7,0.8$, and $1 \mathrm{~mm}$. Measurements and simulations of the output laser fluence showed that the matched spot size could be adjusted with the thickness of the solid nitrous oxide layer.
\end{abstract}

DOI: 10.1103/PhysRevAccelBeams.24.091301

With the expanding number of petawatt-class laser systems [1-3] and recent advances in laser plasma accelerators (LPAs) [4-6], there is an increased interest in using novel accelerating structures as a source of energetic particles for applications including high-energy physics, free-electron lasers, and sources of $\mathrm{THz}$ and $\mathrm{x}$-ray radiation [7-14]. For many of these applications, optical guiding is required to keep the laser intense over extended distances. Without any form of guiding, the interaction length is typically limited by diffraction to the Rayleigh length $Z_{R}=\pi w_{0}^{2} / \lambda$, where $w_{0}$ is the laser spot size and $\lambda$ is the laser wavelength.

To counteract laser diffraction and propagate a pulse over many Rayleigh lengths, thus increasing the final particle beam energy, various waveguides have been developed [15-21]. In a gas-filled capillary discharge waveguide, a discharge pulse heats and ionizes gas inside the channel to form a plasma which develops a radial temperature gradient as heat flows from the hot plasma center to the cold capillary wall [22-30]. This temperature gradient produces a density distribution with an approximately parabolic radial profile of the form $n_{e}(r)=n_{e}(0)+n_{e}^{\prime \prime}(0) r^{2} / 2$. A low-power transversely Gaussian laser pulse can propagate

\footnotetext{
*ajgonsalves@lbl.gov
}

Published by the American Physical Society under the terms of the Creative Commons Attribution 4.0 International license. Further distribution of this work must maintain attribution to the author(s) and the published article's title, journal citation, and DOI. with constant spot size and intensity if the input laser spot size equals the matched spot size

$$
w_{m}=\left(\frac{2}{\pi r_{e} n_{e}^{\prime \prime}(0)}\right)^{1 / 4}
$$

where $r_{e}$ is the classical electron radius. If the input spot size is not matched to the channel, the spot size will oscillate as the laser propagates. In a capillary discharge waveguide, once the plasma reaches equilibrium, the matched spot size scales with the average density $n_{e}$ and capillary radius $r_{\text {cap }}$ as $w_{m} \propto n_{e}^{-1 / 4} r_{\text {cap }}^{1 / 2}$ [28].

The maximum particle energy gain achievable in a single laser plasma accelerator stage scales with the plasma density as $n_{e}^{-1}$. Guiding of pulses with peak intensity of $5 \times 10^{18} \mathrm{~W} / \mathrm{cm}^{2}$ has been demonstrated in a 9-cm long capillary discharge waveguide with a plasma density of approximately $7 \times 10^{17} \mathrm{~cm}^{-3}$, accelerating electron beams up to $4.2 \mathrm{GeV}$ [31]. Further increases in beam energy can be achieved by decreasing the plasma density. However, a smaller capillary radius is required to maintain matched guiding at a lower density, leading to damage as the wings of the laser pulse interact with the channel entrance and walls. Instead, a channel with a larger radius can guide a laser pulse if the depth of the density profile $n_{e}^{\prime \prime}(0)$ is increased. By coupling a nanosecond-scale laser pulse into a capillary discharge waveguide, the plasma can be additionally heated through inverse-bremsstrahlung absorption [32]. This heating reduces the density on axis and creates a stronger channel capable of guiding pulses with a smaller matched spot size. Guiding of pulses with intensities 
up to $10^{19} \mathrm{~W} / \mathrm{cm}^{2}$ has been demonstrated in a 20-cm long capillary discharge waveguide, accelerating electron beams up to $7.8 \mathrm{GeV}$ with a plasma density of $3 \times 10^{17} \mathrm{~cm}^{-3}$ $[6,33,34]$. However, to further increase the beam energy, a reduction in the plasma density is again required, leaving the capillary susceptible to damage.

In this paper, we report the development and operation of a regenerative, variable-radius, cryogenically-formed waveguide. The design of the waveguide enables in situ adjustment of the channel radius, allowing for improved laser guiding. The ability to regenerate the waveguide allows for a wide range of channel diameters to be explored while alleviating the problem of laser damage, increasing the waveguide lifetime.

The waveguide was a modified 6-cm long and heliumfilled capillary discharge waveguide with a $1-\mathrm{mm}$ diameter channel laser machined into sapphire plates. Two slots $1 \mathrm{~mm}$ in diameter were machined $6 \mathrm{~mm}$ from the ends of the sapphire plates and introduced gas into the central channel. Using liquid nitrogen, the sapphire plates were cooled at a rate of $1.5 \mathrm{~K} / \mathrm{s}$. The temperature was measured by a thermocouple attached to an outside wall of the sapphire. Once the temperature reached $155 \mathrm{~K}$, nitrous oxide $\left(\mathrm{N}_{2} \mathrm{O}\right)$ gas controlled by a pulsed valve was flowed through the gas slots into the central channel for up to $2.2 \mathrm{~s}$ with a backing pressure of $10 \mathrm{psi}$. At this wall temperature, the gas deposited onto the inside wall of the channel, forming a stable shell of solid $\mathrm{N}_{2} \mathrm{O}$.

The thickness and uniformity of the solid $\mathrm{N}_{2} \mathrm{O}$ shell along the length of the center of the channel, between the gas slots, was measured by a Thorlabs spectraldomain optical coherence tomography system with a $5 \mu \mathrm{m}$ axial resolution. The capillary was imaged using two $f=200 \mathrm{~mm}, 2$ in. diameter achromatic lenses. A schematic of the setup is shown in Fig. 1(a).

In this design, longitudinally uniform solid $\mathrm{N}_{2} \mathrm{O}$ shells were achieved only at a wall temperature of $155 \pm 2 \mathrm{~K}$. At a higher temperature, the nitrous oxide gas would not deposit onto the channel wall. In addition, because the entire sapphire plate was cooled, deposition also occurred inside the gas slots. At a wall temperature much lower than $155 \mathrm{~K}$, gas deposited inside the slots at a much faster rate than inside the central channel which resulted in longitudinally nonuniform shells and eventually blockage of the slots.

The duration of the $\mathrm{N}_{2} \mathrm{O}$ gas flow through the capillary as set by the opening time of the pulsed valve determined the thickness of the solid layer: the longer the duration of the gas flow, the thicker the solid $\mathrm{N}_{2} \mathrm{O}$ shells (see Supplemental Materials [35]). The inset in Fig. 1(b) shows an example longitudinal measurement of the shell thickness between the gas slots for a valve opening time of $1.5 \mathrm{~s}$, resulting in a $111 \pm 7.5 \mu \mathrm{m}$ thick shell. The thickness is defined as a moving average over successive $60 \mu \mathrm{m}$ increments to smooth out small-scale fluctuations in the
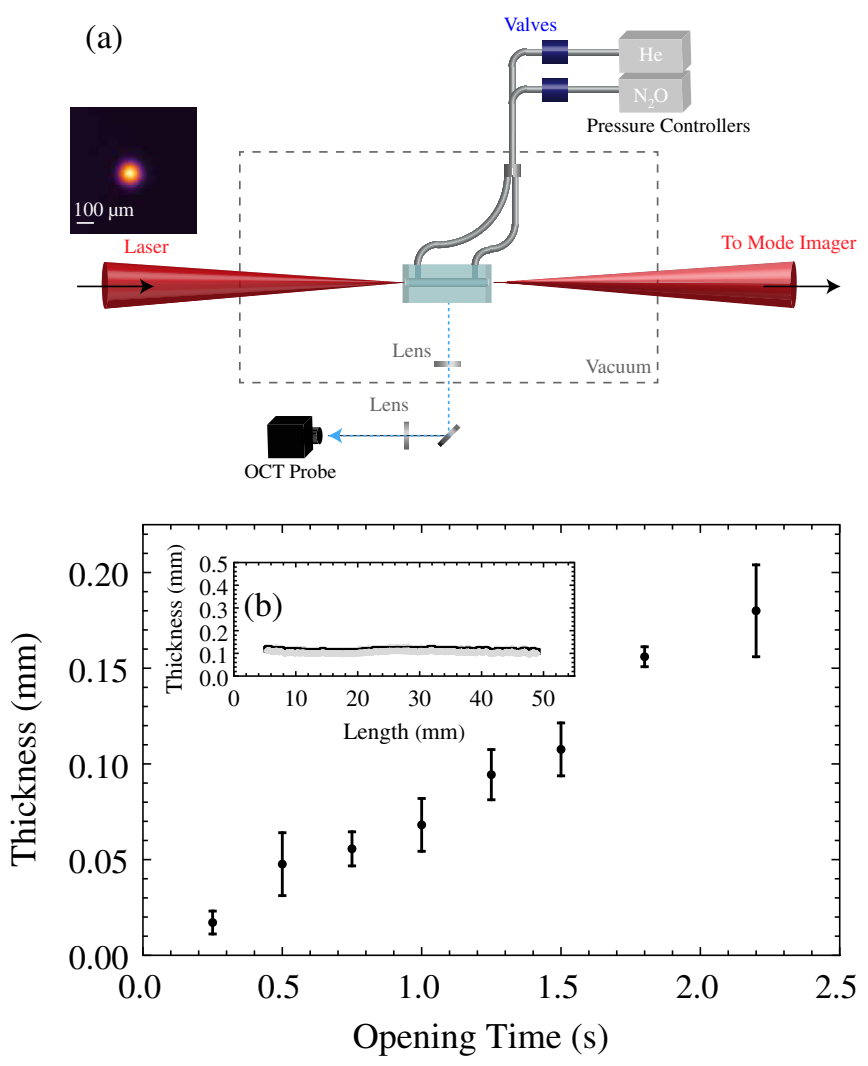

FIG. 1. (a) Schematic of the laser guiding and optical coherence tomography system setup. (b) Average thickness of the solid $\mathrm{N}_{2} \mathrm{O}$ shell as a function of the pulsed valve's opening time. The error bars correspond to fluctuations in the measured thickness as a result of surface roughness. The inset shows the longitudinal thickness between the gas slots for an opening time of $1.5 \mathrm{~s}$. The gray shaded region represents fluctuations in the measured thickness as a result of surface roughness.

thickness caused by surface roughness. By adjusting the opening time of the pulsed valve from 0.25 to $2.2 \mathrm{~s}$, solid shells with thicknesses varying from 50 to $180 \mu \mathrm{m}$ were reproducibly deposited [see Fig. 1(b)]. The $\mathrm{N}_{2} \mathrm{O}$ shell thickness increased at a rate of $67 \mu \mathrm{m} / \mathrm{s}$ the pulsed valve was open. The maximum shell thickness was limited by deposition inside the gas slots which prevented gas flow into the central channel. Reduced heat transfer between the gas and the sapphire through improvements such as thermally isolating the gas slots can help mitigate this problem, allowing thicker shells and smaller channel diameters to be realized.

Once the solid $\mathrm{N}_{2} \mathrm{O}$ layer formed, the capillary was filled with 20 Torr of helium gas. Two stainless steel electrodes at each end of the capillary, separated from the sapphire by approximately $0.5 \mathrm{~mm}$ to prevent a decrease in the electrode temperature, provided a voltage differential along the length of the capillary. A $17-\mathrm{kV}$ pulser system caused the helium gas to breakdown, and a damped, approximately sinusoidal current pulse flowed through the capillary with 
500 A maximum current and $320 \mathrm{~ns}$ quarter period. The discharge evenly ablated the solid $\mathrm{N}_{2} \mathrm{O}$ at a rate of approximately $50 \mathrm{~nm} / \mathrm{shot}$ for the thicknesses tested. Allowing more $\mathrm{N}_{2} \mathrm{O}$ gas to flow through the channel enabled further deposition and regeneration of the solid shell.

In order to understand the behavior of the plasma density profile inside a capillary discharge waveguide with solid $\mathrm{N}_{2} \mathrm{O}$ walls, we performed magnetohydrodynamic simulations using the $1 \mathrm{D}$ code NPINCH [28]. To correctly model the capillary with a solid layer of $\mathrm{N}_{2} \mathrm{O}$, the description of the $\mathrm{N}_{2} \mathrm{O}$ layer as well as the phase transition from condensed to gaseous $\mathrm{N}_{2} \mathrm{O}$ were incorporated. The phase transition onsets were set at a spinodal curve, when the matter becomes dynamically unstable, with thermodynamically unstable states included in the equation of state. Only the first stage ionization of helium was included with a maximum ionization level of seven for nitrous oxide. The description of the $\mathrm{N}_{2} \mathrm{O}$ layer included only the inner $10 \mu \mathrm{m}$, assuming that deeper layers were not affected significantly during a single shot simulation. The boundary was set to have no motion with a temperature of $100 \mathrm{~K}$ such that the resulting $\mathrm{N}_{2} \mathrm{O}$ vapor pressure matched the experimentally measured vapor pressure of 0.1 Torr. The region inside the internal diameter was at first occupied by helium with a density of $4.32 \mu \mathrm{g} / \mathrm{cm}^{3}$. The discharge was defined by the boundary condition for the azimuth component of the magnetic field, which required the total electric current through the capillary to be of the form $I(t)=I_{0} \sin \left(\pi t / t_{0}\right)$, where $I_{0}=500 \mathrm{~A}$ and $t_{0}=650 \mathrm{~ns}$.

From Fig. 2, we can see the evolution of the plasma density during the discharge for a $100 \mu \mathrm{m}$ shell thickness. In a manner similar to the case with no solid $\mathrm{N}_{2} \mathrm{O}$ present, a parabolic density profile forms as the helium is heated and ionized by the electrical current $[28,36]$. However, at approximately $100 \mathrm{~ns}$ before the peak of the current the ablation of the $\mathrm{N}_{2} \mathrm{O}$ layer starts. A fraction of the ablated material forms a cold, dense, and almost neutral gas layer of about $22 \mu \mathrm{m}$ thick on top of the shell. This layer has $T_{e} \gg$ $T_{i}$ and is slightly ionized (the degree of ionization varies from $10^{-4}$ to 0.1 ). The ablated $\mathrm{N}_{2} \mathrm{O}$ plasma then propagates inward, pushing helium toward the capillary axis and increasing in the plasma density. However, the helium plasma maintains an approximately parabolic shape near the axis. By the end of the current pulse, approximately $130 \mathrm{~nm}$ of the solid $\mathrm{N}_{2} \mathrm{O}$ layer is ablated, approaching the experimentally measured ablation rate considering a fraction of the nitrous oxide will redeposit. The $\mathrm{N}_{2} \mathrm{O}$ plasma extends up to $150 \mu \mathrm{m}$ into the channel, and at the helium$\mathrm{N}_{2} \mathrm{O}$ interface, the plasma temperature is approximately $3 \mathrm{eV}$ with an ionization level of 1.8. Since diffusion of different ion species is not included in the model, the interface between helium and $\mathrm{N}_{2} \mathrm{O}$ is a sharp discontinuity. NPINCH simulations were performed for 0 (no solid $\mathrm{N}_{2} \mathrm{O}$ ), 100, and $150 \mu \mathrm{m}$ shell thicknesses. Using the plasma

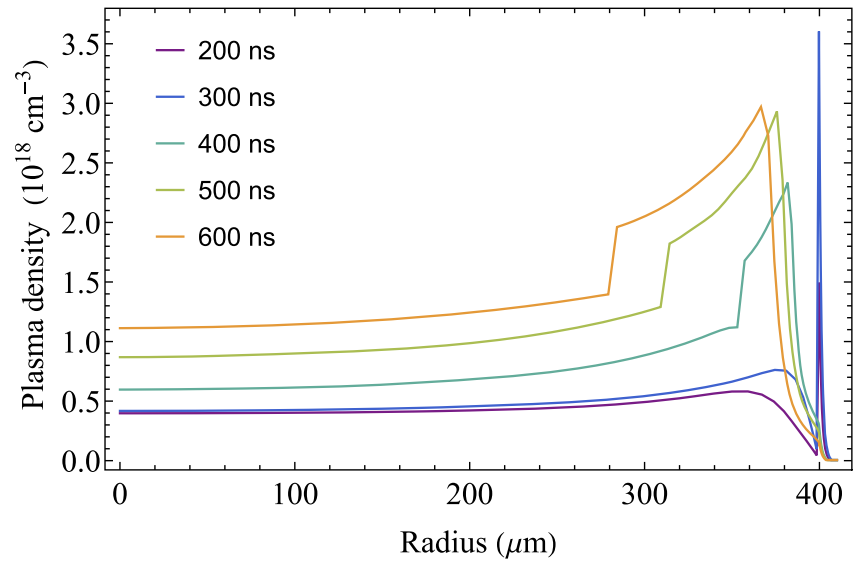

FIG. 2. Simulated radial density profiles for various times in the discharge. Discontinuities in the density are seen after $250 \mathrm{~ns}$, corresponding to the contact discontinuity at the interface between the helium and $\mathrm{N}_{2} \mathrm{O}$ plasmas.

density profile obtained in these simulations, the matched spot size was calculated for each of these channels [37]. The on axis densities and matched spot sizes are shown in Fig. 3. When no solid $\mathrm{N}_{2} \mathrm{O}$ is present, the density is nearly uniform after the peak of current, as expected from the quasistatic equilibrium shown in previous work [28]. Correspondingly, the matched spot size is approximately constant. In contrast, in the channels with solid $\mathrm{N}_{2} \mathrm{O}$ layers, a continuous rise in density, and thus a continuous decrease in matched spot size, is observed after the peak of the discharge current as ablated $\mathrm{N}_{2} \mathrm{O}$ compresses the helium inside the channel. In this case, the equations for the matched spot size and density as given in Ref. [28] are not appropriate, and the scaling of the matched spot size is determined by the motion of the ablated solid $\mathrm{N}_{2} \mathrm{O}$-helium interface.

After $350 \mathrm{~ns}$, near the peak of the discharge current, the densities in the 0 and $100 \mu \mathrm{m}$ cases are almost identical while the matched spot sizes are 118 and $100 \mu \mathrm{m}$, respectively, demonstrating some tunability in the matched

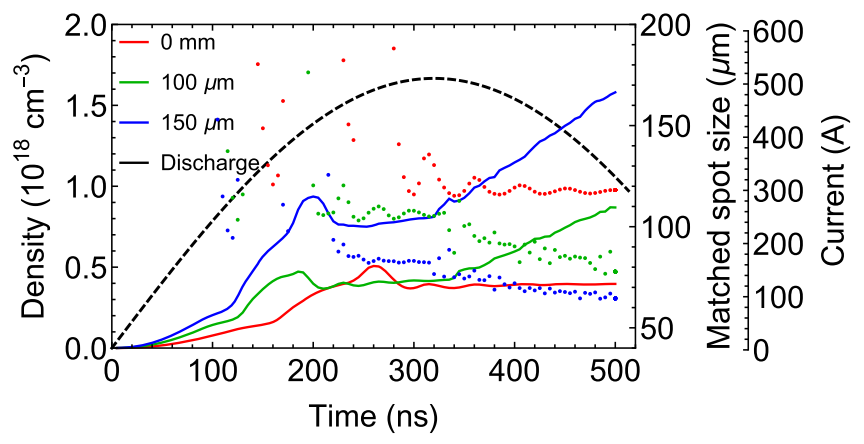

FIG. 3. Temporal evolution of the current (black, dashed line), as well as on axis density (colored lines) and matched spot size (colored points) for solid $\mathrm{N}_{2} \mathrm{O}$ layers of thickness 0 (red), 100 (green), and $150 \mu \mathrm{m}$ (blue). 
spot size as a result of the $\mathrm{N}_{2} \mathrm{O}$. It should be noted that for the current used, the helium is only singly ionized, and for guiding laser pulses above the ionization threshold of the second level, it would be advantageous to either increase the current or replace the helium with hydrogen.

To study the guiding properties of the plasma channel, low-power laser pulses were propagated through the capillary. Guiding of pulses was performed using a fraction of the $1 \mathrm{~Hz}$ repetition rate, Ti:sapphire-based BELLA petawatt laser [38], providing pulses with $785 \mathrm{~nm}$ central wavelength, $1 \mathrm{~ns}$ full width at half maximum (FWHM) pulse duration, and $4 \mathrm{~nJ}$ on target. As shown in Fig. 1(a), the laser pulses were focused using an $f=1.68 \mathrm{~m}$ lens into the entrance of the capillary to an $87 \mu \mathrm{m}$ FWHM beam waist for an intensity of $33 \mathrm{~kW} / \mathrm{cm}^{2}$. Laser transmitted through the capillary was imaged onto a charge-coupled device which could be translated along the beam axis to image either the entrance or the exit plane of the capillary.

Solid $\mathrm{N}_{2} \mathrm{O}$ shells with 100 and $150 \mu \mathrm{m}$ thicknesses were formed and compared to the case with no solid $\mathrm{N}_{2} \mathrm{O}$. $\mathrm{N}_{2} \mathrm{O}$ was redeposited onto the walls of the channel every 200 shots, corresponding to a $10 \mu \mathrm{m}$ change in thickness over the lifetime of the shell. The arrival time between the laser pulse and the discharge current was varied, and Fig. 4(a) shows representative images of the transmitted laser mode at the exit of the capillary. When the laser pulse was injected before and early in the discharge, the output spot size was large. For later times in the discharge, the spot size decreased as the plasma channel formed and guided the pulse.

The normalized peak fluence, defined as the ratio of the peak fluence at the exit plane of the capillary and at the focal plane, for the different solid $\mathrm{N}_{2} \mathrm{O}$ shell thicknesses was measured near the peak of the discharge. Figure 4(b) shows the normalized peak fluences as well as the channel matched spot sizes. In the case of no solid $\mathrm{N}_{2} \mathrm{O}$, the matched spot size could be retrieved from measurements of laser centroid oscillations [39]. For the cases with solid $\mathrm{N}_{2} \mathrm{O}$, the low-capillary temperature introduced significant temporal jitter in the experiment that rendered that technique time-consuming. Therefore, the matched spot size was retrieved by modeling the beam propagation using the 2D cylindrical code INF\&RNO [40,41]. The measured laser mode, retrieved from its vacuum propagation using a Gerchberg-Saxton algorithm, was propagated through parabolic plasma channels of different matched spot sizes. By matching the simulated laser fluences with the experiment, the matched spot sizes of the channels were obtained, and the results are also shown in Fig. 4(b).

In order to check the validity of this method, the matched spot size for the case of no solid $\mathrm{N}_{2} \mathrm{O}$ was also retrieved using the laser centroid oscillation technique. The results obtained by the two techniques were equal to within the experimental error of $\pm 3 \mu \mathrm{m}$. When the laser spot size approaches the matched spot size, the output fluence is very sensitive to the channel and thus the matched spot size.

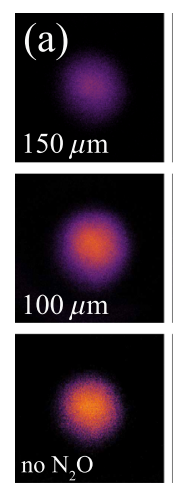

$-130 \mathrm{~ns}$
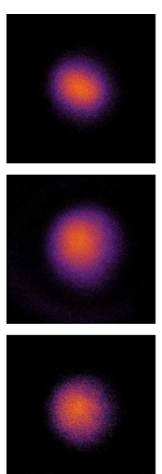

$60 \mathrm{~ns}$
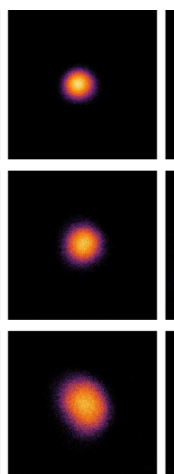

$430 \mathrm{~ns}$
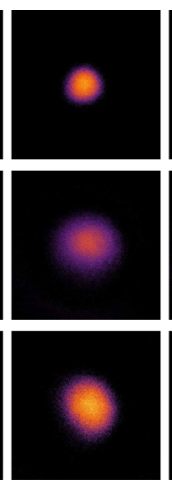

$560 \mathrm{~ns}$
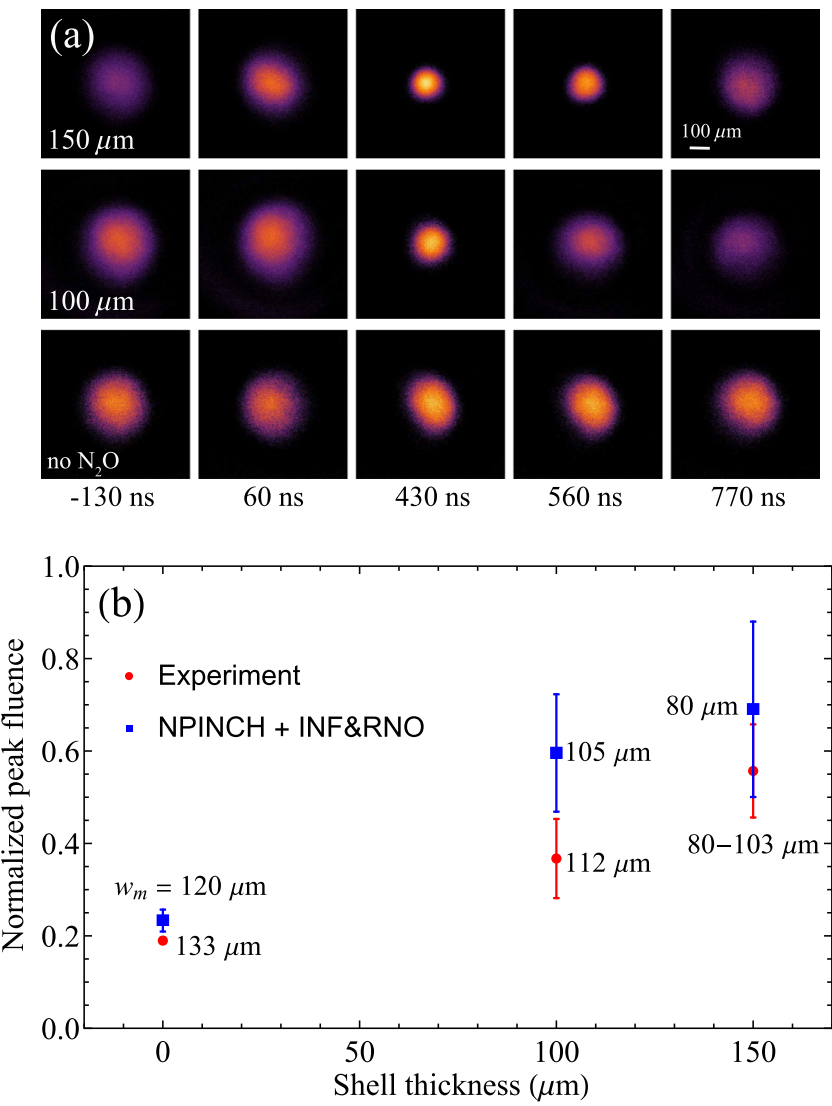

FIG. 4. (a) Representative images of the laser output mode at the exit of the capillary for 150,100 , and $0 \mu \mathrm{m}$ shell thicknesses for discharge delays of $-130,60,430,560$, and $770 \mathrm{~ns}$. Image intensities have been normalized to the maximum intensity for that diameter. (b) Output fluence measured in the experiment (red) and simulated using the experimentally measured matched spot size (blue) as a function of shell thickness. Data were averaged for delays between $20 \mathrm{~ns}$ before and $60 \mathrm{~ns}$ after the peak of the discharge pulse. The error bars correspond to the standard deviation of the data, and the labels represent the matched spot sizes $w_{m}$. In the case of no solid $\mathrm{N}_{2} \mathrm{O}$, the experimental normalized peak fluence has an error of 0.006 .

Therefore, there is significant ambiguity in the matched spot size in the $150-\mu \mathrm{m}$ thick shell because of the experimental fluctuations in peak fluence. Nevertheless, it is clear that the matched spot size is reduced for increasing ice thickness as predicted in Fig. 3.

In addition, INF\&RNO simulations were performed using the NPINCH density profiles and the experimentally measured laser mode. The results as shown in Fig. 4(b) demonstrate the same trend: the matched spot size decreases as the shell thickness increases. For thicker shells, simulations using the NPINCH density profiles predict higher fluences and lower matched spot sizes relative to the parabolic density profile. Further work is required to understand the differences between the two approaches which may be due to the assumptions of one-dimensional symmetry, approximations of the equations of state, or errors in 
the knowledge of the initial experimental conditions. The error bars are the standard deviation of the matched spot sizes shown in Fig. 3 for times between 20 ns before and $60 \mathrm{~ns}$ after the peak of the discharge where there is larger variability in the matched spot size.

Here, we presented the development of a new waveguide formed by flowing $\mathrm{N}_{2} \mathrm{O}$ gas through a capillary with walls cooled to a temperature below the freezing temperature of the gas. The nitrous oxide deposited onto the walls of the capillary, forming a stable, solid shell whose thickness was controlled by the duration of the $\mathrm{N}_{2} \mathrm{O}$ gas flow. Channels with longitudinally uniform shell thicknesses from 50 to $180 \mu \mathrm{m}$ were reproducibly formed. Helium flowing through the channel facilitated discharge and an approximately transverse parabolic plasma density profile developed as was corroborated by simulations and experiments. We verified in situ adjustment of the waveguide's matched spot size by forming solid $\mathrm{N}_{2} \mathrm{O}$ shells with varying thicknesses and detailed a technique for regenerating ablated layers.

This waveguide has several important features for applications that require guiding of laser pulses. In a discharge capillary, the matched spot size is controlled using the channel radius and on axis density. To reach higher particle beam energies, lower plasma densities are required which, for a fixed laser spot size, necessitates a smaller channel radius. However, the capillary then becomes more susceptible to laser damage [31]. With the ability to redeposit solid $\mathrm{N}_{2} \mathrm{O}$ onto the capillary walls, the cryogenic waveguide could operate with a longer lifetime. Additionally, the design allows for some independent control of the on axis density inside the channel using the helium gas pressure and control of the channel radius using the flow duration of the $\mathrm{N}_{2} \mathrm{O}$ gas. Through coordination of these two parameters, better matching between the channel radius and the laser spot size could be achieved for improved guiding in a variety of different regimes. Future work will examine whether this cryogenic waveguide can be applicable as a hollow or near-hollow plasma channel.

The authors gratefully acknowledge for their technical contributions Don Syversrud, Zach Eisentraut, Dave Evans, Mark Kirkpatrick, Art Magana, Greg Mannino, Joe Riley, Nathan Ybarrolaza, and Csaba Toth. This work was supported by the Director, Office of Science, Office of High Energy Physics, of the U.S. Department of Energy under Contract No. DE-AC02-05CH11231, the NSF under Grant No. PHY-1415596, and the project High Field Initiative (Grant No. CZ.02.1.01/0.0/0.0/15 003/ 0000449) from the European Regional Development Fund.

[1] M. Perry, D. Pennington, B. Stuart, G. Tietbohl, J. Britten, C. Brown, S. Herman, B. Golick, M. Kartz, J. Miller,
H. Powell, M. Vergino, and V. Yanovsky, Opt. Lett. 24, 160 (1999).

[2] C. L. Haefner et al., in Society of Photo-Optical Instrumentation Engineers (SPIE) Conference Series, Society of Photo-Optical Instrumentation Engineers (SPIE) Conference Series Vol. 10241 (International Society for Optics and Photonics, 2017), p. 1024102.

[3] C. N. Danson, C. Haefner, J. Bromage, T. Butcher, J.-C. F. Chanteloup, E. A. Chowdhury, A. Galvanauskas, L. A. Gizzi, J. Hein, D. I. Hillier et al., High Power Laser Sci. Eng. 7, e54 (2019).

[4] T. Tajima and J. M. Dawson, Phys. Rev. Lett. 43, 267 (1979).

[5] E. Esarey, C. B. Schroeder, and W. P. Leemans, Rev. Mod. Phys. 81, 1229 (2009).

[6] A. J. Gonsalves et al., Phys. Rev. Lett. 122, 084801 (2019).

[7] C. B. Schroeder, E. Esarey, C. G. R. Geddes, C. Benedetti, and W. P. Leemans, Phys. Rev. ST Accel. Beams 13, 101301 (2010).

[8] D. Jaroszynski, R. Bingham, E. Brunetti, B. Ersfeld, J. Gallacher, B. van der Geer, R. Issac, S. Jamison, D. Jones, M. de Loos, A. Lyachev, V. Pavlov, A. Reitsma, Y. Saveliev, G. Vieux, and S. Wiggins, Philos. Trans. R. Soc., A 364, 689 (2006).

[9] W. P. Leemans, C. G. R. Geddes, J. Faure, C. Tóth, J. van Tilborg, C. B. Schroeder, E. Esarey, G. Fubiani, D. Auerbach, B. Marcelis, M. A. Carnahan, R. A. Kaindl, J. Byrd, and M. C. Martin, Phys. Rev. Lett. 91, 074802 (2003).

[10] J. van Tilborg, C. B. Schroeder, C. V. Filip, C. Tóth, C. G. R. Geddes, G. Fubiani, R. Huber, R. A. Kaindl, E. Esarey, and W. P. Leemans, Phys. Rev. Lett. 96, 014801 (2006).

[11] A. Rousse, K. T. Phuoc, R. Shah, A. Pukhov, E. Lefebvre, V. Malka, S. Kiselev, F. Burgy, J.-P. Rousseau, D. Umstadter, and D. Hulin, Phys. Rev. Lett. 93, 135005 (2004).

[12] V. Shpakov et al., Phys. Rev. Lett. 122, 114801 (2019).

[13] J. van Tilborg, S. Steinke, C. G. R. Geddes, N. H. Matlis, B. H. Shaw, A. J. Gonsalves, J. V. Huijts, K. Nakamura, J. Daniels, C. B. Schroeder, C. Benedetti, E. Esarey, S. S. Bulanov, N. A. Bobrova, P. V. Sasorov, and W. P. Leemans, Phys. Rev. Lett. 115, 184802 (2015).

[14] R. Pompili et al., Phys. Rev. Lett. 121, 174801 (2018).

[15] C. G. Durfee and H. M. Milchberg, Phys. Rev. Lett. 71, 2409 (1993).

[16] S. Jackel, R. Burris, J. Grun, A. Ting, C. Manka, K. Evans, and J. Kosakowskii, Opt. Lett. 20, 1086 (1995).

[17] M. Borghesi, A. J. Mackinnon, R. Gaillard, O. Willi, and A. A. Offenberger, Phys. Rev. E 57, R4899 (1998).

[18] F. Dorchies, J. R. Marquès, B. Cros, G. Matthieussent, C. Courtois, T. Vélikoroussov, P. Audebert, J. P. Geindre, S. Rebibo, G. Hamoniaux, and F. Amiranoff, Phys. Rev. Lett. 82, 4655 (1999).

[19] B. Cros, C. Courtois, G. Malka, J. R. Matthieussent, G. Marques, F. Dorchies, F. Amiranoff, S. Rebibo, G. Hamoniaux, N. Blanchot, and J. Miquel, IEEE Trans. Plasma Sci. 28, 1071 (2000).

[20] W. P. Leemans, B. Nagler, A. J. Gonsalves, C. Tóth, K. Nakamura, C. G. R. Geddes, E. Esarey, C. B. Schroeder, and S. M. Hooker, Nat. Phys. 2, 696 (2006). 
[21] C. Mcguffey, M. Levin, T. T. Matsuoka, V. Chvykov, G. Kalinchenko, P. Rousseau, V. Yanovsky, A. Zigler, A. Maksimchuk, and K. Krushelnick, Phys. Plasmas 16, 113105 (2009).

[22] Y. Ehrlich, C. Cohen, A. Zigler, J. Krall, P. Sprangle, and E. Esarey, Phys. Rev. Lett. 77, 4186 (1996).

[23] D. Kaganovich, P. V. Sasorov, Y. Ehrlich, C. Cohen, and A. Zigler, Appl. Phys. Lett. 71, 2925 (1997).

[24] D. Kaganovich, A. Ting, C. I. Moore, A. Zigler, H. R. Burris, Y. Ehrlich, R. Hubbard, and P. Sprangle, Phys. Rev. E 59, R4769 (1999).

[25] S. M. Hooker, D. J. Spence, and R. A. Smith, J. Opt. Soc. Am. B 17, 90 (2000).

[26] M. C. Marconi, C. H. Moreno, J. J. Rocca, V. N. Shlyaptsev, and A. L. Osterheld, Phys. Rev. E 62, 7209 (2000).

[27] D. J. Spence and S. M. Hooker, Phys. Rev. E 63, 015401 (R) (2000).

[28] N. A. Bobrova, A. A. Esaulov, J.-I. Sakai, P. V. Sasorov, D. J. Spence, A. Butler, S. M. Hooker, and S. V. Bulanov, Phys. Rev. E 65, 016407 (2001).

[29] D. J. Spence, A. Butler, and S. M. Hooker, J. Opt. Soc. Am. B 20, 138 (2003).

[30] A. Butler, D. J. Spence, and S. M. Hooker, Phys. Rev. Lett. 89, 185003 (2002).

[31] W. P. Leemans, A. J. Gonsalves, H.-S. Mao, K. Nakamura, C. Benedetti, C. B. Schroeder, C. Tóth, J. Daniels, D. E. Mittelberger, S. S. Bulanov, J.-L. Vay, C. G. R. Geddes, and E. Esarey, Phys. Rev. Lett. 113, 245002 (2014).
[32] N. A. Bobrova, P. V. Sasorov, C. Benedetti, S. S. Bulanov, C. G. R. Geddes, C. B. Schroeder, E. Esarey, and W. P. Leemans, Phys. Plasmas 20, 020703 (2013).

[33] A. J. Gonsalves et al., Phys. Plasmas 27, 053102 (2020).

[34] C. V. Pieronek, A. J. Gonsalves, C. Benedetti, S. S. Bulanov, J. van Tilborg, J. H. Bin, J. H. Bin, K. K. Swanson, J. Daniels, G. Bagdasarov, N. Bobrova, V. Gasilov, G. Korn, P. Sasorov, C. G. R. Geddes, C. B. Schroeder, W. P. Leemans, and E. Esarey, Phys. Plasmas 27, 093101 (2020).

[35] See Supplemental Material at http://link.aps.org/ supplemental/10.1103/PhysRevAccelBeams.24.091301 for further discussion of deposition process.

[36] G. A. Bagdasarov, P. V. Sasorov, V. A. Gasilov, A. S. Boldarev, O. G. Olkhovskaya, C. Benedetti, S. S. Bulanov, A. Gonsalves, H.-S. Mao, C. B. Schroeder, J. van Tilborg, E. Esarey, W. P. Leemans, T. Levato, D. Margarone, and G. Korn, Phys. Plasmas 24, 083109 (2017).

[37] C. Benedetti, C. Schroeder, E. Esarey, and W. Leemans, Phys. Plasmas 19, 053101 (2012).

[38] K. Nakamura, H. Mao, A. J. Gonsalves, H. Vincenti, D. E. Mittelberger, J. Daniels, A. Magana, C. Toth, and W. P. Leemans, IEEE J. Quantum Electron. 53, 1 (2017).

[39] A. Gonsalves, K. Nakamura, C. Lin, J. Osterhoff, S. Shiraishi, C. Schroeder, C. Geddes, C. Toth, E. Esarey, and W. Leemans, Phys. Plasmas 17, 056706 (2010).

[40] C. Benedetti, C. Schroeder, E. Esarey, C. Geddes, and W. Leemans, AIP Conf. Proc. 1299, 250 (2010).

[41] C. Benedetti, C. Schroeder, C. Geddes, E. Esarey, and W. Leemans, Plasma Phys. Controlled Fusion 60, 014002 (2017). 